\title{
Real Time Control of Plasmas and ECRH Systems on TCV
}

J.I.Paley 1), F.Felici 1), J.Berrino 1), S.Coda 1), N.Cruz 2), B.P.Duval 1), T.P.Goodman 1), Y.Martin 1), J.M.Moret 1), F.Piras 1), A.P.Rodriques 2), B.Santos 2), C.A.F.Varandas 2) and the TCV team

1) Ecole Polytechnique Fédérale de Lausanne (EPFL), Centre de Recherches en Physique des Plasmas, Association Euratom-Confédération Suisse, CH-1015 Lausanne, Switzerland.

2) Associação Euratom/IST, Centro de Fusão Nuclear, Instituto Superior Técnico, Av. Rovisco Pais, 1049-001 Lisboa, Portugal.

e-mail contact of main author: james.paley@epfl.ch

\begin{abstract}
Developments in the real time control hardware on TCV paired with the flexibility of plasma shaping and ECRH actuators are opening many opportunities to perform real time experiments and develop algorithms and methods for fusion applications. The ability to control MHD instabilities is particularly important for achieving high performance fusion plasmas and EC is envisaged as a key actuator in maintaining high performance. We have successfully demonstrated control of the sawtooth instability using the EC launcher injection angle to modify the current profile around the $\mathrm{q}=1$ surface. This paper presents an overview of recent real time control experiments on TCV, developments in the hardware and algorithms together with plans for the future.
\end{abstract}

\section{Introduction}

Tokamaks require real time controllers in order to function successfully and at high performance. The plasma shape, current, density and vertical position needs to be maintained and evolved as necessary. In the future this is likely to be extended to the control of MHD instabilities, in particular using actuators such as the electron cyclotron (EC) systems. TCV has recently installed and begun operations with a new generation of hardware in order to develop and test advanced techniques to optimise plasma control. Together with the multi launcher EC system [1], TCV now has a powerful array of tools to develop real time controllers for plasma parameters such as position, shape and instabilities. In previous experiments, we demonstrated control of the plasma current and elongation using the EC power or the launcher injection angle as the actuator [2]. These techniques have been extended to the sawtooth instability - an important demonstration for instability control in future devices such as ITER. This paper is a review of the latest developments in the real time hardware, experiments and algorithms on TCV.

\section{Developments in real time control hardware on TCV}

The original TCV control system consisted of matrix multiplication of signals and a PID controller $[3,4]$. It generates observers from linear combinations of input signals, such as plasma current, vertical position etc., compares them to reference traces and passes the resulting error signals through PID controllers to provide actuator signals. This provides only linear feedback control of the coil currents, plasma current and density. To provide digital, procedural, non-linear control and to increase the number of control channels available, a multi-DSP VME based system [5,6] has been developed in collaboration with Association Euratom/IST to replace the analogue PID controller. A second system has also been developed which provides the ability to process a large number of signals $(\sim 100)$ using DtAcq [7] acquisition modules and PC CPUs. Both systems are described in the subsequent sections. 


\subsection{DSP-based controller}

Due to the wide variety of plasma shapes that can be obtained in TCV (eg elongations of up to 2.8), unique constraints are placed on the vertical instability controller, which operates on coils inside the vessel: it must be able to run with clock rates (ADC acquisition to DAC update) $\sim 100 \mathrm{kHz}$. Control of the external coils (for shape and position) requires a clock rate of $9-12 \mathrm{kHz}$. To meet these specifications, a multi-DSP VME based system was developed. This system consists of several VME cards, each with four Texas Instruments digital signal processors (DSP) and four analogue inputs and outputs. The cards can be combined through a private communication bus to share data at a rate of up to $50 \mathrm{kHz}$ in order to control the shaping coil currents. Also, each VME card may operate as a separate, independent system at up to $\sim 200 \mathrm{kHz}$, one of which is used to drive the internal vertical field coils. The system has been used in initial tests to control TCV plasma discharges, using algorithms which simply replicate the analogue PID controller. More advanced algorithms are now being developed which include improving the performance of the vertical position control.

\subsection{PC-based, multi-channel controller}

The DSP-based system is limited to $\sim 30$ input and output channels, the majority of which are used to control the coil currents. There are many tokamak diagnostics which generate a large array of analogue signals; for example the soft x-ray diagnostics on TCV provide over 200 signals to cover the soft $\mathrm{x}$-ray emission from the whole vacuum vessel. To run, for example, tomographic inversions of the soft $\mathrm{x}$-ray data or real time equilibrium reconstructions hundreds of analogue input channels are required. Many of these diagnostics at TCV use the D-tAcq 196 Compact-PCI (C-PCI) acquisition modules to acquire up to 96 differential channels of 16bit data per card and store data for post-shot analysis. The cards can be combined in a C-PCI crate to provide acquisition of hundreds of channels. These modules also have the capability to send acquired data to the memory of a host $\mathrm{PC}$ in real time (within a few microseconds of a request). The PC can either be embedded on a C-PCI board and slotted into the C-PCI crate master socket or an external desktop PC containing the latest Intel CPUs connected to the crate via a C-PCI bus extender module. The C-PCI PC modules tend to use laptop-class CPUs that have less computational power available for the real time calculation, but have the advantage of reduced latency times to obtain the acquired data in memory and therefore have the fastest potential clock rates. There are 16 16bit analogue outputs on a 'rear transit module' connected to each D-tAcq card. The fastest possible clock rate is $100 \mathrm{kHz}$, using $96 \mathrm{ADC}$ channels and 16 DAC channels. However, the time from ADC acquisition to DAC update is longer due to the latency required to transfer the data from $\mathrm{PC}$ memory to the DAC (a few $\mu \mathrm{s}$ ). Of course faster clocking rates allow less computational time to run algorithms. Using multiple D-tAcq cards to increase the number of channels reduces the maximum possible clocking rate.

Linux is used as the real time PC operating system. The provided D-tAcq drivers suspend all the interrupts to the CPU during the real time process, guaranteeing the CPU availability. Data samples are transferred to/from the DAC/ADC and the host PC memory using Direct Memory Access (DMA). This dedicates the processor to the real time algorithm. We also use the Linux function mlockall() to prevent paging of the RT algorithm to the swap area. 


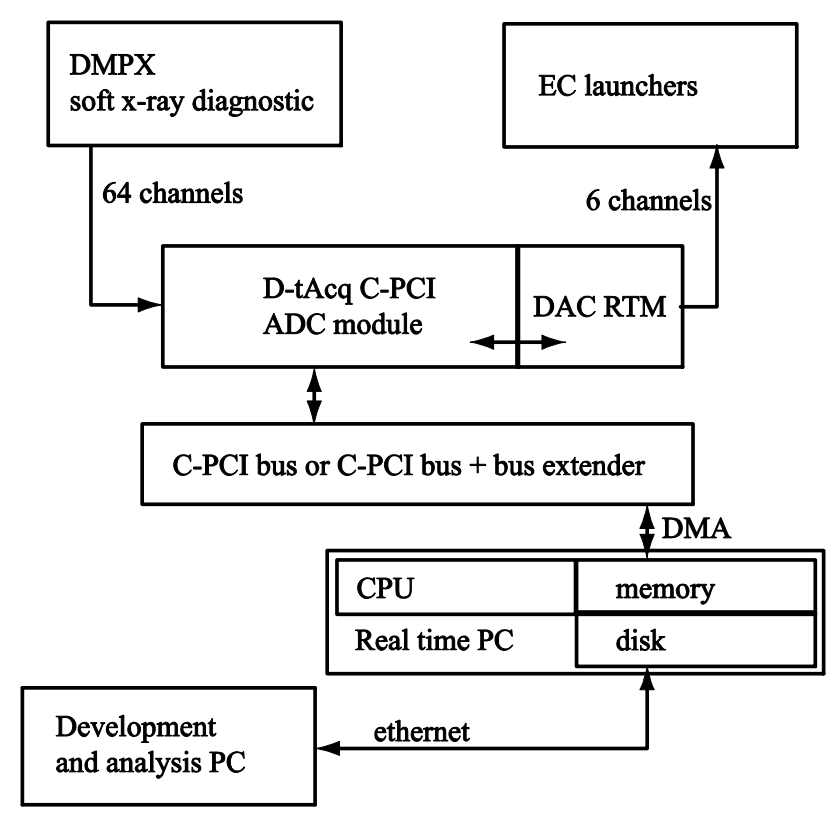

FIG. 1. Block diagram of the D-tAcq real time control system connected to the TCV EC system and $D M P X$ soft x-ray diagnostic.

Simulink ${ }^{\circledR}$ and the real time workshop for embedded targets have been integrated with the DtAcq drivers to facilitate rapid development of algorithms that can then be tested in Simulink against models of the plasma response on a development and analysis PC. The real time workshop generates $\mathrm{C}$ code from the Simulink block diagrams and compiles the code using an Intel compiler for optimum performance (optimised to the specific CPU running the real time PC). The executable is then sent to the real time PC over the network where it can be tested for timing constraints (to ensure the algorithm is completed within each clocking period) before a live test. Several software channels are provided for post-shot debugging as well as timing information and the raw ADC data is stored to allow execution of the Simulink model with the actual acquired data for detailed analysis of the controller algorithms. A block diagram of the system is shown in FIG. 1.

Sawtooth control, profile control, real time equilibrium reconstruction, tomography and shape control are now all under development at TCV using this system.

\subsection{The TCV EC system}

The extensive EC heating and current drive system on TCV, based on $90.5 \mathrm{MW}$ gyrotrons, has the capability of real time control. There are 7 EC launchers, each with independent, real time compatible control of the launcher mirror angle. In this paper we shall focus on the 6gyrotron, 3-MW second-harmonic (X2) subsystem. Each cluster of 3 X2 gyrotrons has a separate power supply, over which we have control of the injected power for each cluster.

\section{Real time control applications and algorithms}

A summary of the first tests using the DSP system to control the coil currents is provided in this section, together with the experiments to control the plasma current and elongation using EC power and launcher actuators. A description of the experiments to control the sawtooth instability in real time using the EC launchers follows, together with the peak in profile control. 


\subsection{Basic control of coil currents using digital PIDs}

The first application of the DSP based controller was to replicate the analogue PID system (which typically only incorporates $\mathrm{P}$ terms and $1 \mathrm{D}$ term). To initialise this project, the PID terms loaded into the analogue system were converted into a transfer function, incorporating all the measured zeros and poles of the analogue circuitry, transformed into discrete time and the resulting coefficients loaded into a digital IIR filter algorithm running in each DSP. The system was able to replicate the analogue PID controller and successfully evolve the coil currents and plasma density though a plasma discharge. Efforts are now underway to optimise this system and develop advanced control algorithms.

\subsection{Real time plasma current and elongation control using EC actuators}

Real time control of the plasma current using EC power was demonstrated in a fully noninductive scenario, where the plasma current is driven entirely by ECCD [8,9]. The plasma current observer was rewired to generate a control signal for the gyrotron power supplies (instead of only the Ohmic coil), using the analogue PID controller (P and I terms only). The resulting controller successfully tracked step changes in the plasma current reference signals [2].

In a constant magnetic quadrupole shaping field, varying the plasma current profile leads to a change in the plasma elongation [10]. By heating off-axis with the ECRH system, the current profile is broadened (by decreasing the resistivity - ECCD has low efficiency off-axis) leading to an elongation of the plasma. A flux observer was used to estimate the real time elongation [11], generate an elongation error signal and actuate the ECRH power to control the elongation.

Where the ECRH beam is deposited above (or below) the plasma midplane and as the plasma elongates, the ECRH deposition becomes more centralised. To maintain the deposition at constant $\rho$, the ECRH mirror launcher angles were controlled in real time. The required mirror angle to maintain the deposition at constant $\rho$ was assumed to be linearly related to the plasma elongation (where the linear relation was deduced from previous plasma discharges by calculating the deposition rho using ray tracing codes.) and programmed using only the $\mathrm{P}$ term of the controller. This was a successful demonstration of launcher tracking techniques which will be required for future MHD instability control.

\subsection{Real time feedback control of the sawtooth instability}

One application of the D-tAcq real time system is to control the sawtooth instability using EC actuators. The sawtooth instability occurs in the plasma core when the plasma current is large enough for a $\mathrm{q}=1$ surface to exist within the plasma. It is a periodic relaxation of the core plasma pressure which is seen in traces of core soft $\mathrm{x}$-ray emission as a slow increase in radiative emission followed by a rapid crash. For large sawteeth, i.e. when the period is very long, the instability may induce a secondary instability, known as a neoclassical tearing modes (NTM) which degrade the plasma confinement and may also cause a disruption - a rapid termination of the plasma discharge $[12,13]$. Sawteeth are also known to remove impurities from the plasma core, which is important for burning plasmas as a build-up of the helium ash may occur in the core, reducing the core reaction rate. For these reasons, it may be necessary to have some control over the sawtooth instability. 
By driving current in the region of the $\mathrm{q}=1$ surface, sawteeth may be destabilised/stabilised as necessary [14]. ECRH is a highly localised heating system that can modify the local resistivity and thus the local current profile. ECCD can also be used to directly inject local current. We therefore have available an actuator to modify the sawtooth period [15]. To calculate the sawtooth period, a sawtooth crash detection algorithm was developed using signals from a multi-chord soft $\mathrm{x}$-ray diagnostic (DMPX). The extracted sawtooth period is subtracted from a target period reference signal to provide a controller error signal.

The plasma response to the launcher angle is non-linear. In a small angular range, the sawtooth response varies rapidly in response to small mirror movements whereas outside this region, there is practically no response [16]. This means the effective controller gain should be large when outside this region and small within. In order to build such controller, it is necessary to have a model of the plasma response on which to test the control algorithm. A model of the sawtooth period response was developed using launcher feedforward sweeps across the $q=1$ surface in both directions (see FIG. 2) to generate a 2D lookup table of the sawtooth periods for the Simulink simulations of the controller. Both directions are necessary to account for hysteresis in the direction of the mirror sweep [17]: sweeping from the core to edge results in a hotter plasma core and a more peaked current profile whereas in the other direction the current profile is flatter. The $\mathrm{q}=1$ surface shifts in each case, due to the change in temperature profile of the plasma and the associated redistribution of plasma current. This shift was taken into account using the second column of the lookup table, taken to be the average position of the mirror over the previous $0.5 \mathrm{~s}$ in time. A density dependence is also present: in these shots the density tends to increase throughout the EC phase, reducing the peak in the sawtooth period.

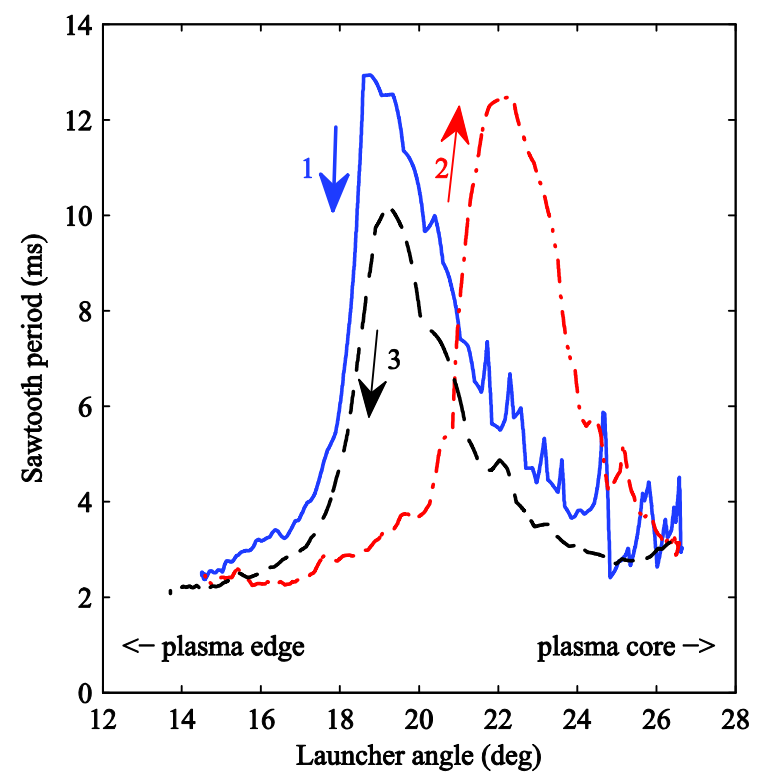

FIG. 2. Feedforward launcher mirror sweeps in the vicinity of the $q=1$ surface for pulse 35807. The launcher is swept 3 times. The first sweep starts with EC deposition inside the $q=1$ surface, towards the plasma core and the launcher angle is reduced until the deposition is outside this surface (the solid blue curve). The $2^{\text {nd }}$ sweep returns the deposition towards the plasma core (dash-dot red curve) and finally the $3^{\text {rd }}$ sweep terminates outside the $q=1$ surface (dashed black curve). The shift in the peak of the sawtooth period demonstrates hysteresis in the sweep due to a redistribution of plasma current. 


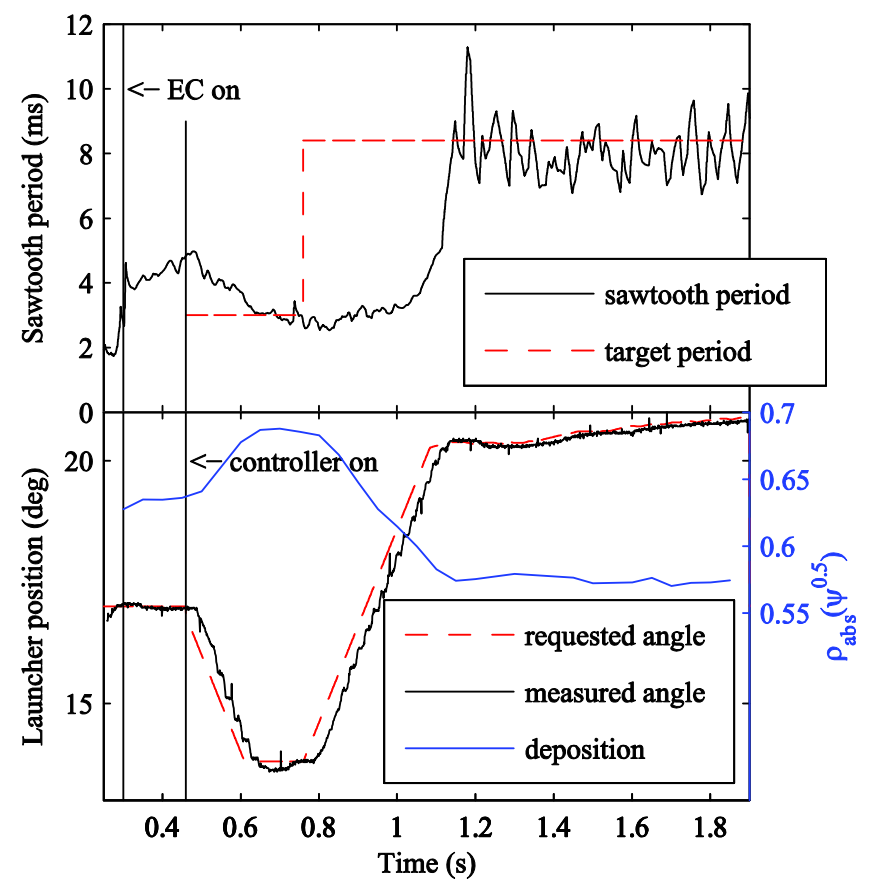

FIG. 3. Real time, closed loop control of the sawtooth period for shot 35833. The target sawtooth period reference signal was initially $3 \mathrm{~ms}$, with a step to $8.5 \mathrm{~ms}$ at $0.75 \mathrm{~s}$. The controller moves the launcher to successfully obtain and track the target. Also shown is the post shot calculation of the deposition in rho of the EC beam.

FIG. 3 shows a demonstration of the sawtooth control. An initial target sawtooth period reference of $3 \mathrm{~ms}$ is set and is followed by a step increase to $8.5 \mathrm{~ms}$. At the start of the controlled phase, there is an initial launcher motion to smaller angle as the sawtooth period is $>3 \mathrm{~ms}$. At the step, the controller rapidly moves the launcher towards the $q=1$ surface, but there is little response until the launcher angle is $\sim 20 \mathrm{deg}$, as expected from FIG. 2. As the observed sawtooth period starts to increase, the controller gain is reduced and the mirror moves more slowly as it tracks the $8.5 \mathrm{~ms}$ reference successfully.

Work is now underway to develop more advanced control algorithms which may use multiple diagnostics to provide, for example, a priori knowledge on the location of the $q=1$ surface. A method of maximising the sawtooth period will also be investigated.

\subsection{Real time profile control}

As a first step towards multi-actuator profile control, a simplified peak-in-profile controller was designed and tested. This used the 64 soft x-ray channels from the DMPX diagnostic and the D-tAcq real time system to track the X-ray emission profile peak. The real-time algorithm determines the maximum of a spline fit to the emission profile. This value is compared to a pre-set target reference value with the resulting error fed to a PI controller and the output to the EC launcher. If the peak value is too high, the controller moves the launcher for more offaxis deposition, while if the peak value is too low, the launcher is oriented to heat more centrally. A preliminary version of the algorithm was successful at tracking a step reference with only small steady-state error (FIG. 4). In a separate experiment, a disturbance was artificially introduced by reducing the gyrotron power. The controller successfully compensated for the decrease in X-ray profile peak by moving the launcher towards the centre. Future experiments will demonstrate control of both the profile peak and shape, using not only the ECRH launcher angles but also the ECRH power. 


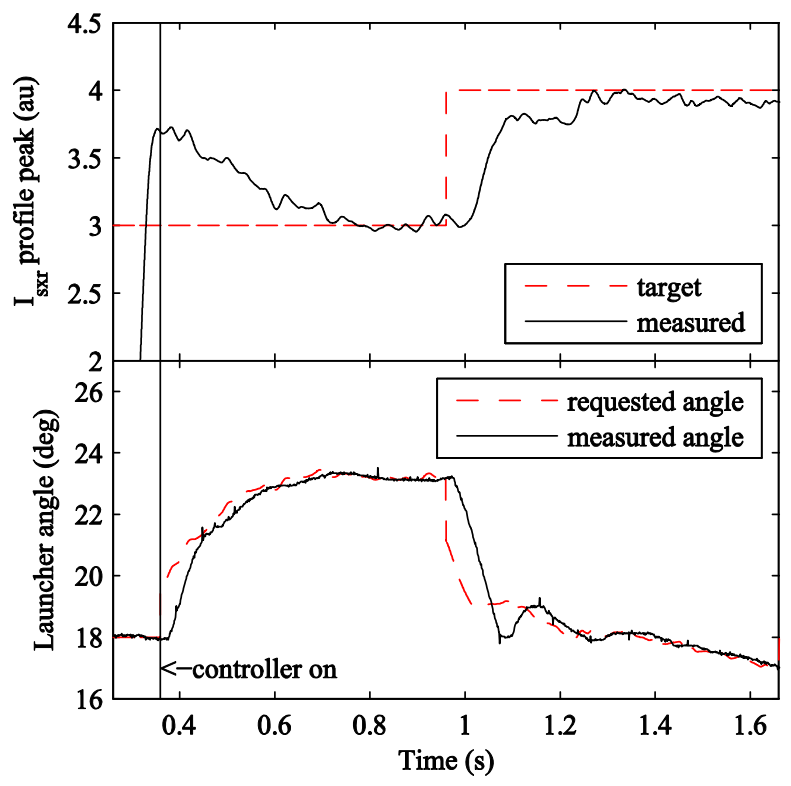

FIG. 4. Real time peak-in-profile control for pulse 35857. The soft $x$-ray profile is fit with a spline in real time and the peak is compared to the target. At the step increase, the controller requested a smaller angle such that the ECRH deposition becomes more centralised and the soft x-ray emission increases.

\subsection{Diagnostic Triggering}

As well as control applications, there are also interesting diagnostics applications for the real time hardware. For example, we have used the D-tAcq real time system to detect the sawtooth crash and generate a trigger signal for a charge exchange recombination spectrometer on $\mathrm{TCV}$. In this way we were able to resolve the evolution of the toroidal plasma rotation during the sawtooth ramp [18], the results of which will be reported upon shortly. Clearly, this is the first of many other diagnostic synchronisation possibilities.

\section{Summary}

EC is a powerful tool for real time control of instabilities. Recent experiments on TCV have provided several demonstrations of real time control using the EC power and launchers.

Coupled with the new control developments on TCV, there are numerous possibilities to test advanced philosophies and algorithms in preparation for ITER and beyond. It is envisaged that EC will be used to control instabilities such as NTMs and sawteeth, by adjusting the launcher mirror angle and EC power. We have used the EC launchers in real time control loops for elongation control and successfully demonstrated control of the sawtooth instability

Further algorithms and applications being developed include NTM, shape, ITB, disruption and temperature control. It is planned to fully integrate the TCV EC system into the central control scheme to allow simultaneous control of both shape and internal plasma properties during a TCV discharge. 


\section{Acknowledgements}

This work was funded by the Euratom Atomic Energy Community under an intra-European fellowship, by Fundação para a Ciência e Tecnologia and in part by the Swiss National Science Foundation.

\section{References}

[1] Goodman, T.P., et al 1996 Proc. 19th SOFT 565

[2] Paley, J.I., et al., Plasma Phys. Control. Fusion 49 (2007) 1735-1746

[3] Lister, J.B., et al., 1997 Fusion Technol. 32321

[4] Isoz, P.F., et al., 1990 Proc. 16th Symp. Fusion Technology (London, UK)

[5] Rodrigues, A.P., et al., Fusion Eng. Des. 60 (2003) 435-441

[6] Rodrigues, A.P., et al., IEEE Transaction on Nuclear Science 53 (2006) 845

[7] D-tAcq Solutions Ltd. http://www.d-tacq.com/

[8] Sauter, O., et al., Phys. Plasmas 8 (2001) 2199

[9] Coda, S., et al., Plasma Phys. Control. Fusion 42 (2000) B311

[10] Pochelon, A., et al., Nucl. Fusion 41 (2001) 1663-9

[11] Hofmann, F., et al., Nucl. Fusion 30 (1990) 2013

[12] Sauter, O., et al., Phys. Rev. Lett. 88 (2002) 105001

[13] Gude, A., et al.,Nucl. Fusion 42 (2002) 833

[14] Sillen, R.M.J., et al., Nucl. Fusion 26 (1986) 303

[15] Angioni, C., et al., Nucl. Fusion 43 (2003) 455-468.

[16] Henderson, M.A., et al., Fusion Engineering and Design 53 (2001) 241-248

[17] Goodman, T.P., et al., FEC IAEA (2002) EX/P5-11

[18] Bortolon, A., et al., private communication. 